\title{
Simulation of dynamic fracture of an impact-loaded brittle solid: microcracked and polycrystalline solids
}

\author{
R W Smith and D J Srolovitz \\ Department of Materials Science and Engineering, The University of Michigan, Ann Arbor, Ml \\ 48109, USA
}

Received 27 February 1995, accepted for publication 10 April 1995

\begin{abstract}
A modified molecular dynamics method is introduced to describe the dynamic fracture and damage accumulation of impact-loaded brittle solids with realistic microstructural features. We examine the influence of pre-existing microcracks and grain boundaries on dynamic fracture. Pre-existing microcracks were found to increase the total damage (pre-existing damage plus accumulated damage due to impact) within a material for all combinations of impact pulse height and microcrack concentration studied. The damage accumulated due to the impact itself, however, is found to increase with increasing concentration of pre-existing microcracks at low impact pulse heights and to decrease with increasing concentration at higher impact levels. Therefore, microcracks may either enhance cracking by providing weaker crack paths or retard it by reffecting and dissipating tensile elastic waves. The presence of weak grain boundaries in a polycrystalline material enhances intergranular fracture while diverting cracks away from grain interiors. Weak grain boundaries lead to the fragmentation of the polycrystalline material into grain size powders. The number of broken grain boundary bonds increases and the number of broken matrix bonds decreases as the grain boundary bond strength is reduced. At low impact pulse strength the total number of broken bonds increases as the grain boundary bonds are weakened, but at high pulse strengths, the total number of broken bonds is reduced as the grain boundary bond strength is reduced. Increasing impact strength shifts the fracture mode from intergranular to transgranular. The principal influence of both microcracks and grain boundaries appears to be their ability to scatter elastic waves propagating through the material.
\end{abstract}

\section{Introduction}

Ceramics and other brittle materials are being employed with increasing frequency in the fabrication of components designed for high-energy impact resistance. Examples of these applications include monolithic ceramics for armour [1] and coatings for turbine blades $[2,3]$, pistons and cylinders $[2,4]$. In these applications, these materials are exposed to impact loads which may compromise material properties by causing immediate catastrophic failure, through impact-induced erosion or by other localized damage accumulation mechanisms. The behaviour of materials in impact-loading situations depends on many intrinsic properties of the materials such as density and elastic moduli. However, once the composition of matter is determined, the impact performance of these materials may be manipulated by changing the microstructure of the material. In the present paper, we examine the influence of microstructure (grain size and grain boundary (GB) toughness) and defect density (microcrack density) on the impact properties of brittle polycrystalline solids.

Over the past decade, several experimental studies have examined the impact behaviour of ceramics and other brittle materials [5-10]. Recently, Gilath et al [5] have shown that 
impact failure in $\mathrm{Al}_{2} \mathrm{O}_{3}$ changes from spallation to Hertzian cone fracture as the area of the impact zone is decreased. Maekawa et al [6] related crack morphology and fracture strength of $\mathrm{SiC}$ to the energy and elastic properties of impinging particles. Ritter and co-workers [7-9] demonstrated that coarse-grained $\mathrm{SiN}$ and $\mathrm{Al}_{2} \mathrm{O}_{3}$ have significantly better impact resistance than fine-grained materials do, and that the resistance in each case increases with increasing chemical purity. Louro et al [10] determined that compressive waves followed by tensile release waves produce significantly more damage than compressive waves alone. They also found that, while the addition of a second phase such as partially stabilized zirconia improves the fracture toughness of statically loaded ceramics [10], such additions have little effect on impact resistance. On the other hand, the presence of second-phase particles containing a large void fraction significantly reduces impact resistance [10]. These results all show that microstructure plays a key role in determining the impact damage resistance of brittle materials.

While these experimental studies have provided important practical information on impact damage and its relation to microstructural and environmental factors, they have not focused on the fundamental mechanisms of the interactions between cracks and microstructural features under impact-loading conditions. In order to investigate impact damage on a more fundamental level and to provide a basis for predicting the properties of different materials, several computer simulation studies of impact loading have been performed. One common simulation approach is based upon the use of hydrocodes (see [11] for an Eulerian hydrocode application to impact). This approach has proven to be successful in applications to metals. However, most applications of this technique contain no information about the microstructure of the material. High rate deformation has also been examined using the finite-element method (FEM) [12]. However, as with the hydrocodes, FEM approaches typically are based upon continuum models of material behaviour and only account for microstructure in an average manner. Although it is possible to include a local description of microstructure and its effects on fracture using these approaches, doing so is not computationally practical because of the fine level of discretization and the frequent remeshing that would be required.

In order to take a more microscopic view of material behaviour, several workers (see, e.g., [13-15]) have applied atomistic simulation methods to predict the properties of materials under impact-loading conditions. In these studies, the material and the projectile are modelled as a small number of atomic planes and a small atomic cluster, respectively. These simulations employed the classical molecular dynamics (MD) method and empirical descriptions of atomic interactions (e.g., Lennard-Jones potentials or embedded-atom method potentials [16]) to simulate both material and projectile. While the MD method has an important advantage over continuum treatments, namely the fact that it need only be parametrized at the atomic level, with no need to put in ad-hoc assumptions regarding the deformation properties of the material, it is greatly limited in the size of the sample and the length of time that can be studied. These constraints have prevented the use of atomistic simulation methods to study impact fracture on a length scale appropriate for examining the role of microstructure in this process.

In a recent paper [17], we introduced an extension to the MD method which is appropriate for examining impact fracture on a microstructural length scale. In the present paper, we employ this method to examine the role played by microstructure in the failure of dynamic impact-loaded brittle materials. The following section reviews the basic structure of the model and describes how it is used to represent various types of microstructure commonly found in ceramics. The results of the application of this method to polycrystalline microstructures of different grain sizes and GB toughnesses are presented. We also apply 
this simulation approach to understand the effects of microcracks on the fracture behaviour of impact-loaded ceramics. Finally, these results are analysed to elucidate the mechanisms by which microstructure modifies the cracking behaviour of dynamically impacted materials.

\section{Method}

The simulation technique which we employ in the present study was originally introduced in [17] and is based upon the MD simulation method. In MD the trajectories of a large number of particles are integrated forwards in time in accordance with Newton's equations of motion. Unlike in traditional atomistic $\mathrm{MD}$, however, we do not identify each particle with an individual atom but rather associate each particle with a small discrete volume of material. The appropriate selection of this discrete volume allows any arbitrarily large region of material to be simulated using a reasonable number of particles. This discretization does, however, imply that no interesting dynamics are occurring on a scale finer than this small volume. In the present simulations, we view the material as being two dimensional and we initially discretize the undeformed material into a regular triangular lattice (i.e. a two dimensional network of equilateral triangles equivalent to the (111) plane of an FCC crystal). The simulation cell is formed by taking a rectangular region of this grid in the $X-Y$ plane spanning 100 particles in the $X$ and 101 particles in the $Y$ directions. In the present study, we discretize the material such that the interparticle spacing is $10 \mu \mathrm{m}$ and the overall dimensions of the simulation cell are $1 \mathrm{~mm}$ in $X$ by $0.86 \mathrm{~mm}$ in $Y$ [17]. The particles on the edges of the sample are left free (i.e. the edges of the sample are free surfaces).

A finite-range pair potential is used to describe the energy of the system. This potential does not describe individual bonds between atoms, but rather the bonding between the particles, which are large groups of atoms. Furthermore, the parameters of the potential are chosen in order to produce the desired elastic properties of the perfect solid. In the present study, we employ the well known Lennard-Jones potential to describe the interparticle interaction. This choice was motivated by the fact that the properties of the Lennard-Jones potential have been exhaustively studied and that it has the qualitatively correct features for describing physical properties on this larger than atomic length scale, i.e. isotropic elastic behaviour at the equilibrium separation and increasing elastic moduli under increasing compression. The Lennard-Jones potential may be written in the following form:

$$
\Phi\left(r_{i j}\right)=A\left(\frac{r_{0}}{r_{i j}}\right)^{12}-B\left(\frac{r_{0}}{r_{i j}}\right)^{6}+C
$$

where $r_{i j}$ is the interparticle separation and $r_{0}, A, B$ and $C$ are constants. $r_{0}$ is determined by our choice of the discretization of the material. $C$ determines the zero of energy, which we unconventionally choose to be at the minimum of the potential. As in the traditional Lennard-Jones potential, $A / B=\frac{1}{2}$ and the value of $A$ (or $B$ ) is chosen by fitting to the bulk modulus of the solid. Values for these parameters for the $\mathrm{Al}_{2} \mathrm{O}_{3}$ potential used in this work are given in table 1 .

Since we are interested in the study of brittle materials, equation (1) must be modified to reflect the fact that 'bonds' between particles break if they are stretched too far. Although bond breaking is a reversible process on the atomic scale, in a more macroscopic view (as considered here) breaking 'bonds' between the larger-than-atomic particles is irreversible. This is because fracture typically creates rough surfaces that will not mate together perfectly if the two pieces of material are brought back into contact and/or a chemical reaction (such as oxidation) may occur such that the surfaces created in fracture are different when the two pieces of material are put back into contact. Therefore, we model the breaking of 
Table 1. Data for $\mathrm{Al}_{2} \mathrm{O}_{3}$ and parametrization of $\phi$.

\begin{tabular}{ll}
\hline Parameter & Value \\
\hline Density & $3.8 \times 10^{3} \mathrm{~kg} \mathrm{~m}^{-2}$ \\
Bulk modulus & $350 \mathrm{GPa}$ \\
Surface energy & $4 \mathrm{~J} \mathrm{~m}^{-2}$ \\
$r_{0}$ & $1.0 \times 10^{-5} \mathrm{~m}$ \\
$r_{C}$ & $1.002 r_{0}$ \\
$A$ & $5.613127 \times 10^{-6} \mathrm{~J}$ \\
$B$ & $11.22625 \times 10^{-6} \mathrm{~J}$ \\
$C$ & $5.613127 \times 10^{-6} \mathrm{~J}$ \\
$m$ & $3.2418 \times 10^{-12} \mathrm{~kg}$ \\
$\tau$ & $1.0 \times 10^{-10} \mathrm{~s}$ \\
\hline
\end{tabular}

interparticle bonds by irreversibly modifying the interparticle potential when the particles are separated by a distance greater than a cut-off distance $r_{c}$. Toward this end, when $r_{i j}$ first exceeds $r_{c}$, the interparticle potential described in equation (1) is truncated beyond $r_{0}$, such that $\Phi\left(r_{i j}\right)=0$ for $r \geqslant r_{0}$. Note that $r_{0}<r_{c}$. Therefore, once a bond has snapped, its ability to support a load in tension is destroyed, although it retains its strength in compression. This condition is expressed in the model via the broken-bond potential which behaves as

$$
\Phi_{B}\left(r_{i j}\right)= \begin{cases}\Phi\left(r_{i j}\right) & r_{i j} \leqslant r_{0} \\ 0 & r_{i j}>r_{0} .\end{cases}
$$

Therefore, there is no resistance to increasing the spacing between particles once the bond is broken, but the resistance to decreasing the spacing between particles (beyond the equilibrium separation $r_{0}$ ) is unchanged. This type of potential prevents two opposing faces of a crack from interpenetrating if a compressive stress is applied. The value of the parameter $r_{c}$ is chosen such that the energy liberated upon breaking a bond is equal to the lattice parameter times twice the surface energy. Therefore, bond breaking in this model occurs when the system satisfies the classical Griffith theory of brittle fracture (i.e. the critical strain energy release rate $G_{c}$ is equal to twice the surface energy).

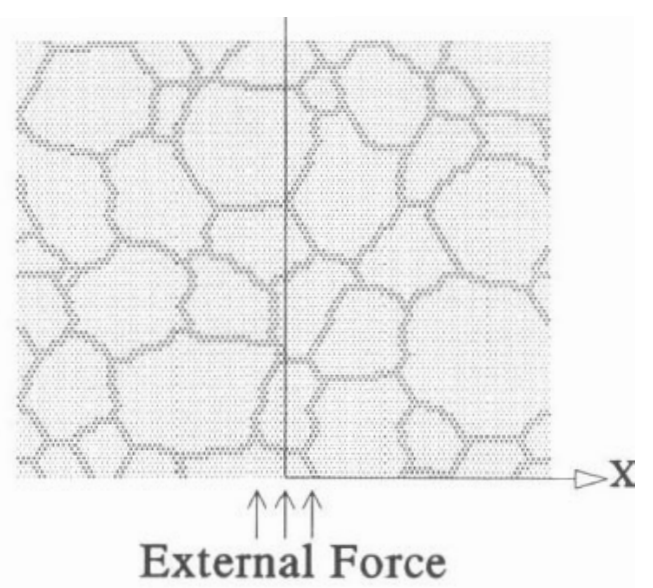

Figure 1. Simulation cell with sample GB structure superimposed onto the triangular lattice. The dark symbols indicate particles lying along the boundary. The arrows indicate the target area for the extemal impact and the impact direction. 
(a)

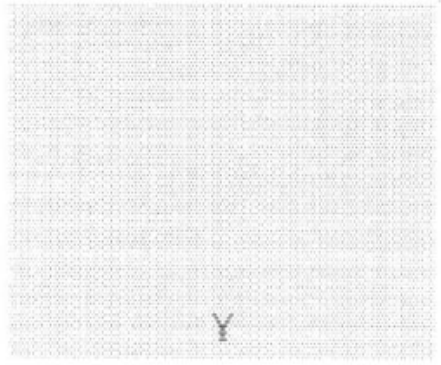

(c)

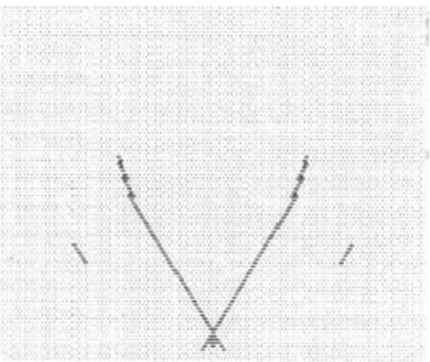

(e)

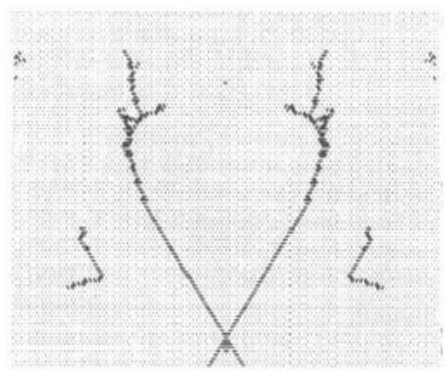

(b) $\mathrm{t}=500 \tau$
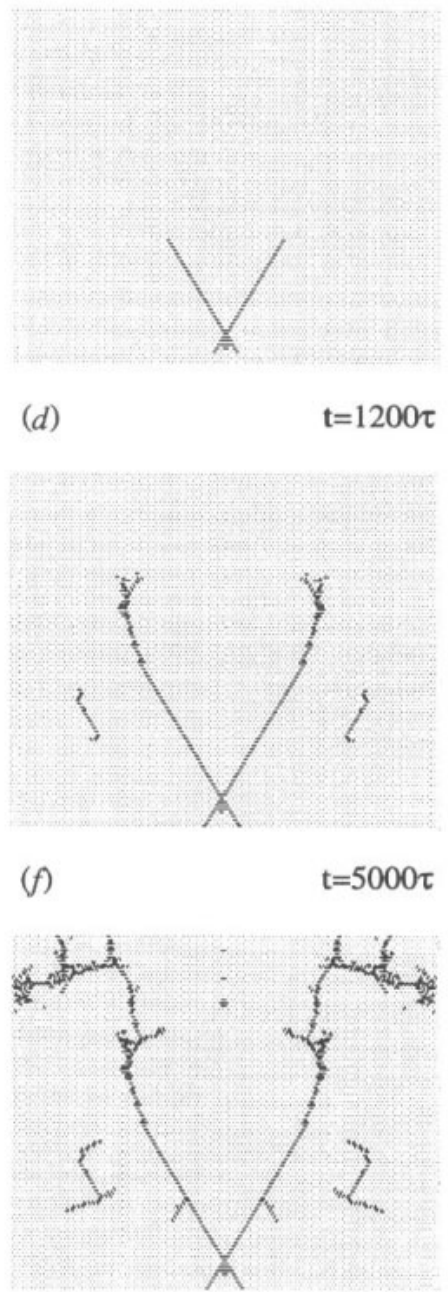

Figure 2. Spatial distribution of broken bonds as a function of time for an initially defect-free sample subjected to an impulse of $F_{\max }=0.375 \mathrm{~N} /$ particle. Lines drawn between particles in each frame indicate bonds that have been broken on or before the time step in each figure. These figures show the initial particle positions (i.e. the displacements associated with the loading are not shown).

The present discretized material model captures most of the important physics associated with the fracture of brittle solids but, at the time, introduces some artificial effects. The current model reasonably describes the elastic behaviour of a two-dimensional solid with a Poisson ratio of $1 / 3$ [18]. Application of this model to the quasi-static propagation of a crack in a uniform material shows that it quantitatively reproduces the Griffith condition for crack propagation [18]. The distribution of stresses around a fibre consisting of bonds of different stiffness is in excellent correspondence with the predictions of isotropic continuum elastic theory (solved numerically) [19]. A random distribution of broken bonds [20] leads to a failure distribution which is well represented by the Weibull distribution, which is 
commonly used to describe the failure distribution in brittle ceramics. On the other hand, the discreteness of the model introduces some artifacts. For example, the elastic field around a crack is not divergent in the present model: the divergence is cut-off at a length scale comparable to the interparticle separation. This would cause some difficulties if we were focussing on the growth of short cracks-introducing artificially high values of the fracture toughness. In such situations, the present model should be viewed only as semiquantitative. This model is also somewhat artificial in that there are no energy dissipation mechanisms (e.g., dislocation motion, grain boundary sliding) other than the fracture process itself: real materials always show at least some dissipation. While this introduces artifacts when comparing the present results with real materials, it also simplifies the problem to a degree that it allows the study of the fundamentals of dynamic fracture without undue interference from extraneous effects. This is appropriate since non-bond-breaking energy dissiaption mechanisms vary in strength by many orders of magnitude between different 'hrittla' motarinla

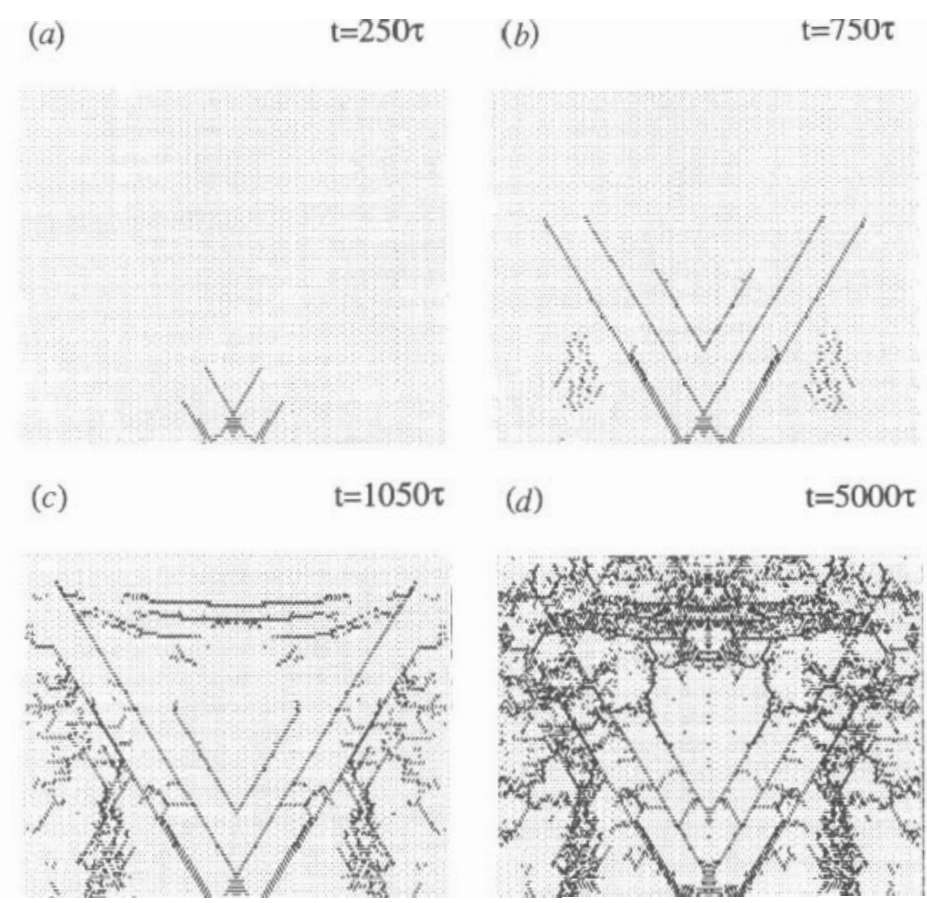

Figure 3. Spatial distribution of broken bunds as a function of time for an initially defect-free sample subjected to an impulse of $F_{\max }=0.750 \mathrm{~N} /$ particle.

The use of a larger-than-atomic particle size and the interparticle potential (described above) provides a framework for describing any type of microstructure in a manner appropriate for a dynamic fracture simulation. One may simply superimpose the desired microstructure on the initial regular lattice of particles. The microstructural features may be accommodated by appropriate choice of the parameters $r_{0}, r_{c}$ and $A$ for each bond in the system. Bonds in the matrix region are assigned constants $\left(r_{0}, r_{c}\right.$ and $\left.A\right)$ appropriate to the matrix material and bonds lying in regions of other phases or spanning interfaces may be similarly tuned with different constants to reflect the behaviour of these regions or interfaces. Elastically stiff or soft precipitates may be modelled by adjusting the magnitude 
of $A$. The toughness of an interface may be modified by changing the value of the fracture cut-off distance $r_{c}$. Misfitting inclusions may be modelled by adjusting the equilibrium interparticle distance $r_{0}$. Features such as microcracks are handled simply by breaking bonds prior to beginning the simulation. In the current work, we have simulated the presence of pre-existing microcracks by breaking randomly selected bonds before the application of the impact load. We have also modelled a polycrystalline microstructure by including a network of GBs for which the fracture cut-off distance $r_{c}$ is reduced relative to that in the matrix (figure 1).

Once the microstructure is discretized into an array of particles, the simulation is started by applying an external impact pulse to pre-selected particles at an external surface of the sample. In this study, the external force $F$ is applied in the direction normal to the surface. This external force rises linearly from $F=0$ to $F=F_{\max }$ over a duration of 250 time steps and then decreases back to $F=0$ over the next 250 steps (i.e. a triangular pulse). Forces are applied in the positive $Y$ direction to the centre ten particles on the $Y=0$ line of particles (see figure 1). Response to the impact is studied by integrating Newton's equations of motion for each particle forwards in time for a total of 5000 time steps of duration $1.0 \times 10^{-10} \mathrm{~s}$.

\section{Results}

The dynamic impact simulation method, described above, has been applied to samples representing a perfect microstructureless material, a randomly microcracked material and a polycrystalline material (i.e. with a network of GBs). During each simulation, particle positions and velocities and the state of each bond (broken or unbroken) were monitored at each time step.

\subsection{Perfect material}

Although we have considered the evolution of damage in an initially defect-free material in a previous publication [17], we review those results in order to compare with the present simulations which are focused on the influence of microstructure and/or defects on dynamical fracture of impact-loaded materials. An example of crack propagation in an initially defectfree sample during impact loading with a relatively small impact pulse height is shown in figure 2 for the $F_{\max }=0.375 \mathrm{~N} /$ particle simulation. The first two frames show that the crack begins in a small central region eight lattice spacings ahead of the centre of the impact zone and quickly bifurcates into a small $\mathrm{X}$-shaped crack pattern. The crack radiates forwards into the material and backwards towards the free surface, where the impact occurred. By the third frame (figure $2(\mathrm{c})$ ), two new independent cracks have nucleated on either side of the $\mathrm{X}$ crack and begin to grow parallel to the branches of this original crack. The direction of propagation of the main $X$-crack branches then begins to shift towards the vertical (i.e. impact direction), and then additional crack branching occurs (see figure $2(d)$ ). At late times, new cracks begin to form near the top corners of the sample and grow towards the main cracks as the simulation proceeds (see figures $2(e)$ and $2(f)$ ).

Figure 3 shows the results of a simulation performed with a much stronger maximum force $F_{\max }=0.750 \mathrm{~N} /$ particle. In this case the initial $\mathrm{X}$ crack is immediately flanked by two additional cracks running parallel to the branches of the $\mathrm{X}$ (figure $3(a)$ ). These cracks initiate on the impact surface at the interfaces between the loaded region and the unloaded regions. As these cracks propagate into the material, two independent areas of damage nucleate and grow near the bottom corners of the sample (figure $3(b)$ ). Finally, a large 
damage region forms near the top end of the sample between the branches of the initial $\mathrm{X}$ crack (figure $3(c)$ ). From this point in time, bonds continue to break at various positions throughout the material at a rate that decreases steadily with time.

The evolution of the number of broken bonds during the simulations depicted in figures 2 and 3 above are shown in figures $4(a)$ and $4(b)$, respectively. For the low-impact simulation, bonds begin to break at $t=250 \tau$ (figure $2(a)$ ), which corresponds to the time where the applied force reaches its maximum. Following an initially high damage rate, the rate at which bonds break slows until $t=950 \tau$, where it suddenly accelerates. This acceleration corresponds to the appearance of the first two independent cracks, outside the $\mathrm{X}$ crack (figure 2(c)). After the nucleation and growth of these cracks, the damage rate once again decreases, yielding a plateau in the number of broken bonds versus time, which extends to $t=2500 \tau$. At this time step, the damage rate again increases as additional independent cracks nucleate and begin to grow in the vicinity of the upper corners of the sample (figure 2(e)). After the formation of these cracks, the damage accumulation rate slows again and a second plateau is formed which extends to the end of the simulation.

In the high-impact simulation, bonds begin to break at $t=200 \tau$, slightly before the applied load has reached its maximum, and damage is accumulated at a nearly constant rate until $t=750 \tau$. At this time, the diffuse damage regions form in the bottom corners of the sample (figure $3(b)$ ) and the damage rate accelerates. At $t=1000 \tau$ the damage rate accelerates again as cracks are formed near the top of the sample in a concave pattern between the branches of the $\mathrm{X}$ crack (figure $3(c)$ ). Following the appearance of these cracks, the damage rate decreases smoothly until the end of the simulation.

Table 2. Number of runs at each test condition for microcrack simulations.

\begin{tabular}{lcccccc}
\hline \multirow{2}{*}{$\begin{array}{l}\text { Number of broken } \\
\text { bonds (\%) }\end{array}$} & \multicolumn{6}{c}{ Number of runs for the following $F_{\max }$ in N/Particle } \\
\cline { 2 - 7 } & 0.2500 & 0.3125 & 0.3750 & 0.5000 & 0.6250 & 0.7500 \\
\hline 0 & 1 & 1 & 1 & 1 & 1 & 1 \\
1 & & & 188 & & & 25 \\
3 & & & 60 & & & 25 \\
6.5 & 10 & 10 & 20 & 10 & 10 & 35 \\
10 & & & 20 & & & 25 \\
\hline
\end{tabular}

\subsection{Microcracked material}

Microcracks were introduced into the samples by randomly breaking a pre-determined number of nearest-neighbour bonds prior to the start of the simulations. A single broken bond cannot accurately model a true microcrack because such a defect does not exhibit the requisite elastic singularities. Nonetheless, broken bonds do represent significant stress concentrators and a distribution of broken bonds is a reasonable, albeit qualitative, description of distributed fine-scale damage. Simulations were performed on samples containing pre-broken bonds in concentrations of $1 \%, 3 \%, 6.5 \%$ and $10 \%$. Impact pulse heights ranged from $F_{\max }=0.25$ to $0.75 \mathrm{~N} /$ particle and were applied in the positive $Y$ direction to the ten particles in the middle of the $Y=0$ line of particles. For statistical significance, each simulation was repeated between 20 and 188 times using a different random distribution of initially broken bonds. Table 2 presents the matrix of test conditions 
(a)

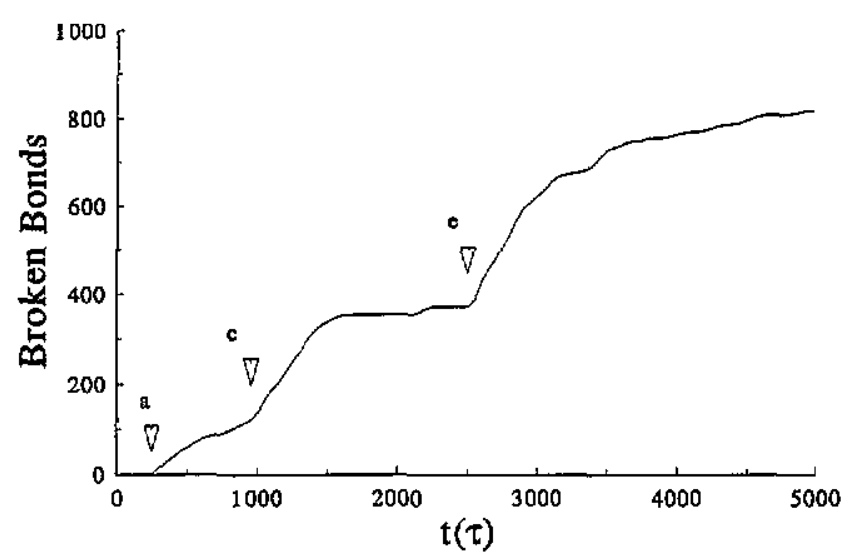

(b)

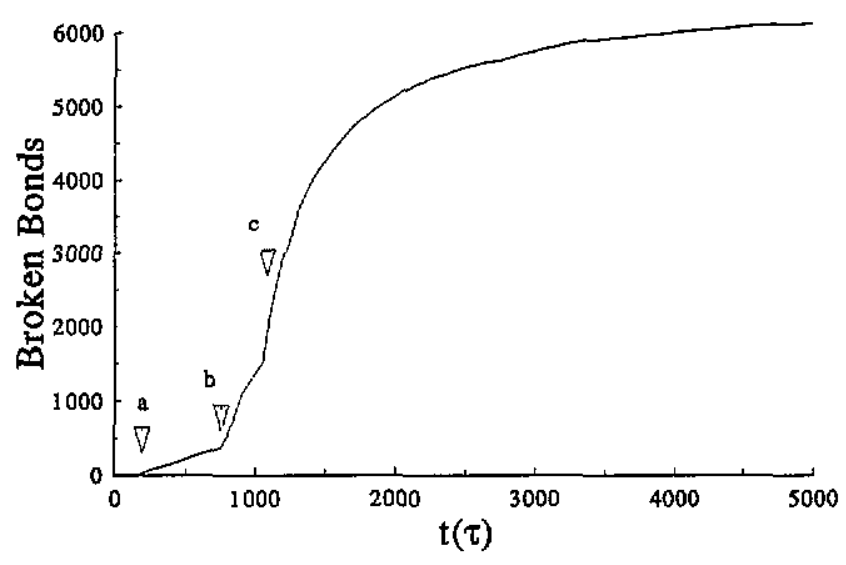

Figure 4. Number of broken bonds versus time for initially defect-free samples: $(a) F_{\max }=$ $0.375 \mathrm{~N} /$ particle (see figure 2); (b) $F_{\max }=0.750 \mathrm{~N} /$ particle (see figure 3).

employed and the number of independent simulations that were used in each case. A total of $444 \mathrm{MD}$ simulations were performed.

A representative distribution of broken bonds at the end of a simulation made with an initial defect concentration of $1 \%$ and an impact pulse height $F_{\max }=0.375 \mathrm{~N} /$ particle is shown in figure 5. The most apparent effect of the pre-existing microcracks on the simulations is the loss of symmetry (in the $X$ direction) in the crack nucleation and growth. This crack morphology should be compared with that shown in figure $2(f)$, which corresponds to the same loading but of an initially defect-free sample. The central X-shaped crack, common to most defect-free material simulations [17], appears in the present, initially microcracked case as well. However, unlike in the defect free case, one of the branches of the $X$ fails to extend. Additional independent cracks nucleate at sites of pre-broken bonds and some of the cracks which nucleate in the initially defect free case do not form in the initially microcracked samples. Simulations performed at higher initial defect concentrations 


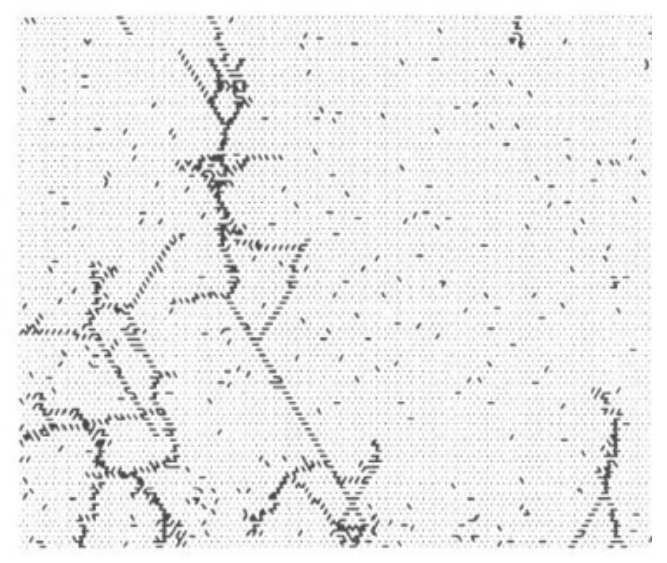

Figure 5. Representative distribution of broken bonds at the end of the simulation for a sample containing an initial microcrack concentration of $1 \%$ subjected to an impact of $F_{\max }=0.375 \mathrm{~N} / \mathrm{particle}$.

show a similar, although more pronounced deviation trom the crack behaviour observed in the initially perfect material.

The dependence of the number $N_{d}$ of bonds broken during the simulation and the total number $N_{l}$ of broken bonds (including pre-broken bonds) on the initial microcrack density are shown in figure $6(a)$ for simulations performed using an impact pulse height $F_{\max }=0.375 \mathrm{~N}$ /particle. $N_{d}$ increases very slowly with increasing initial microcrack concentration and, hence, $N_{t}$ is approximately equal to the initial microcrack concentration. Interestingly, at the higher impact pulse height $\left(F_{\max }=0.75 \mathrm{~N} /\right.$ particle), the number of bonds that break during a simulation $N_{d}$ monotonically decreases with increasing initial microcrack density (see figure 6(b)). Surprisingly, the total number $N_{t}$ of broken bonds at the end of the simulations shows a minimum with respect to the initial microcrack density.

The numbers $N_{d}$ and $N_{t}$ of broken bonds both increase as functions of the impact pulse height in the simulations conducted on both the initially defect-free sample and the samples containing a non-zero density of initial microcracks (figure 7). For each of the pulse heights investigated, $N_{t}$ is larger for the pre-cracked samples. For pulse heights less than $F_{\max }=0.50 \mathrm{~N} /$ particle, $N_{d}$ is also greater in the pre-cracked samples (with an initial microcrack density of $6.5 \%$ ), but for larger pulse heights the initially perfect sample accumulates more damage during the simulation than the pre-cracked samples.

The results in figures 6 and 7 show that the total number of broken bonds increases with increasing initial defect concentration for almost all values of $F_{\max }$ examined. The number of bonds broken during the simulation, however, increases with increasing initial microcrack density at low $F_{\text {max }}$ and decreases with increasing initial damage concentration at higher $F_{\text {max }}$. In order to investigate the mechanisms by which the initial damage affects the number of bonds broken during the simulations, it is instructive to compare the time dependence of damage accumulation for the initially perfect and pre-cracked materials. Figure $8(a)$ shows $N_{d}$ as a function of time for samples subjected to the high impact pulse height $F_{\max }=0.750 \mathrm{~N} /$ particle. Near the beginning of the simulations $(t<1000 \mathrm{t})$, the behaviours of $N_{d}(t)$ are similar for all defect concentrations. The curves for the precracked samples begin to deviate from that of the initially defect-free material only after the pronounced acceleration in damage rate at $t \approx 1100 \tau$. Following this rapid damage accumulation, the rate of damage accumulation slows more quickly as the initial microcrack density increases. Therefore, it is in the late stages of damage accumulation where the initial microcrack density plays an important role at high impact pulse height.

For the simulations performed at the lower impact pulse height $F_{\max }=0.375 \mathrm{~N} /$ particle, 
(a)

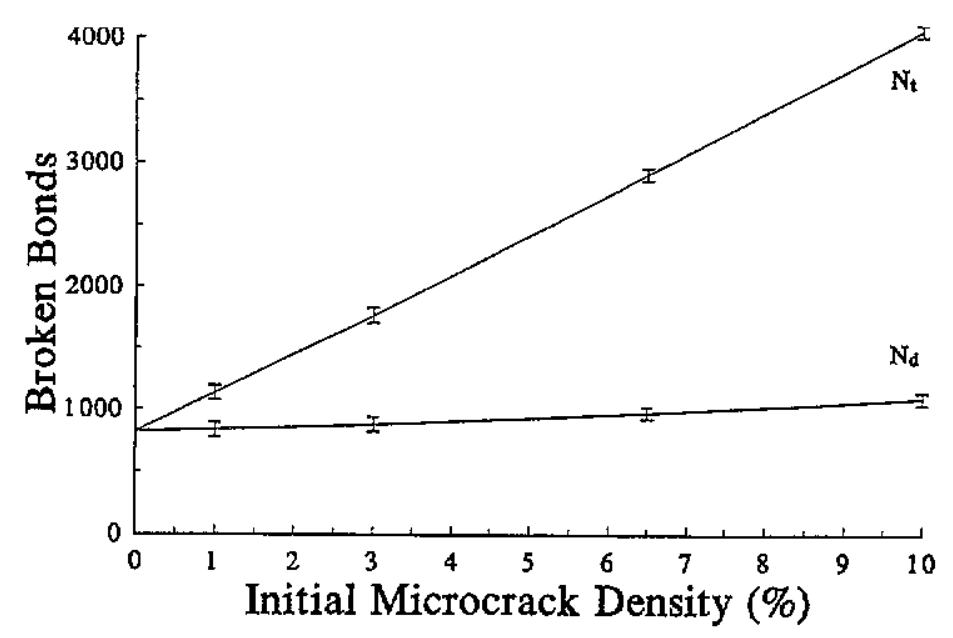

(b)

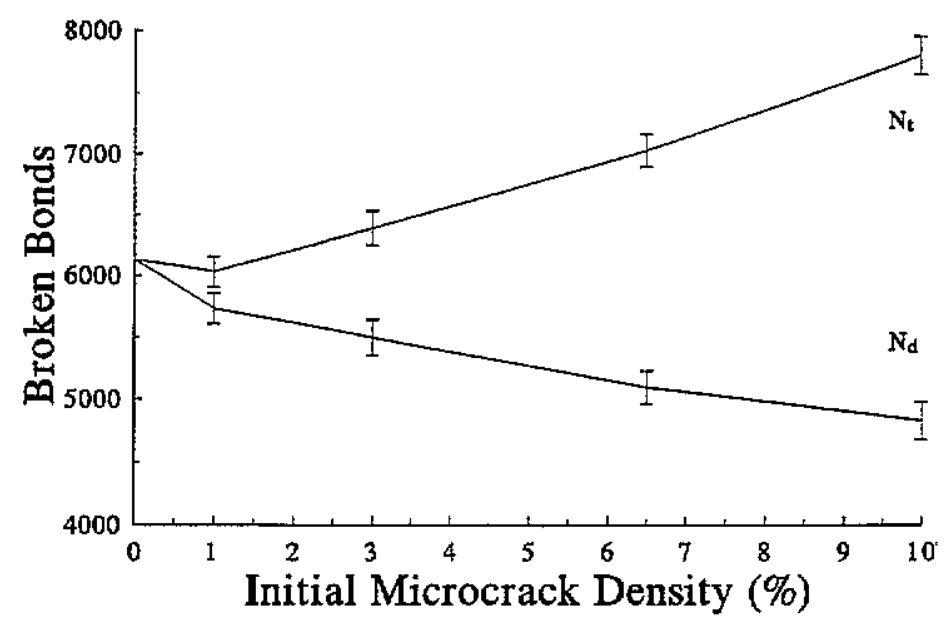

Figure 6. Number of bonds broken during simulation and total number of broken bonds (including pre-broken bands) versus initial defect concentration: (a) $F_{\max }=0.375 \mathrm{~N} / \mathrm{particle;}$ (b) $F_{\max }=0.750 \mathrm{~N} /$ particle. Error bars show $\pm 1 \sigma$.

figure $8(b)$ shows that the behaviour of the pre-cracked samples differs from that of the initially defect-free material from the very beginning of the simulations. The pre-cracked samples display a higher damage rate prior to $t=1000 \tau$ and the damage rate increases with increasing initial defect concentration. The main features of the $N_{d}$ versus time plot for the initially defect-free sample (i.e. the abruptness of the acceleration in damage accumulation at $t=1000 \tau$, the subsequent plateau and the second acceleration at $t=2500 \tau$ ) are increasingly smoothed as the initial microcrack density is increased. The increase in the final values of $N_{d}$ with increasing initial defect concentration results principally from the increased damage rate exhibited by the pre-cracked samples during the early phase of the simulations. Therefore, it is the early stage of damage accumulation where the initial 


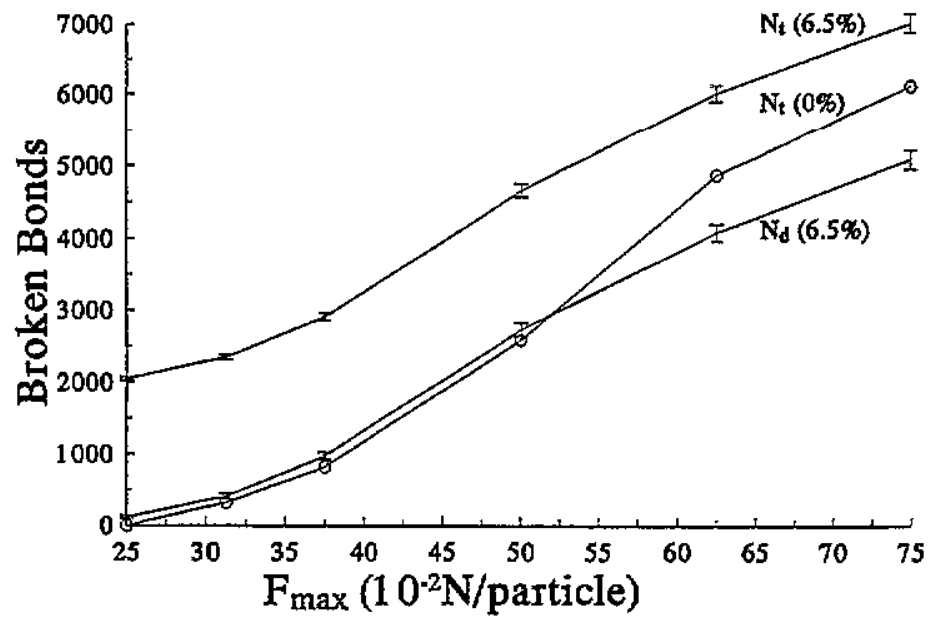

Figure 7. Number of bonds broken during simulation and total number of broken bonds (including pre-broken bonds) versus impact pulse height for simulations made with initially perfect material and material containing an initial defect concentration of $6.5 \%$. Error bars show $\pm 1 \sigma$.

microcrack density plays an important role at low impact pulse heights.

\subsection{Polycrystalline material}

Several simulations were performed in which normal grain growth polycrystalline microstructures were superimposed onto the lattice as depicted in figure 1. The GB network acts to partition the particles into specific grains. The GBs lie between nearest-neighbour sites in adjacent grains. The particular polycrystalline microstructures employed here were generated with a Monte Carlo grain growth algorithm described in [21]. The GBs are distinguished from the grain interiors by modification of the $G B$ bond properties. In the present simulations, the GB bonds were weakened relative to the matrix by shortening the length $r_{c}$ to which the bonds could be stretched before breaking. The parameters controlling the bond stiffness and bond length (i.e. $A, B$ and $r_{0}$ ) were not changed from the grain interior bond values (although these parameters could be modified as well). Simulations were performed in which the maximum extension $r_{c}^{g b}$ of GB bonds was chosen such that the maximum energy supported in tension by these bonds, namely $E_{g b}=\Phi\left(r_{c}^{g b}\right)$, ranged from 0.01 to 1.0 of that supported by the matrix bonds, $E_{m a t}=\Phi\left(r_{c}\right)$. This corresponds to a GB toughness 0.01 to 1.0 times that of the grain interior. In each simulation, an impact pulse height between $F_{\max }=0.25$ and $0.75 \mathrm{~N} /$ particle was applied to the central ten nodes of the $Y=0$ line of nodes. Two grain sizes $\left(R / r_{0}=7\right.$ and 15$)$ were investigated and in each case the simulations were repeated ten times using different microstructures (of the same grain size) in order to generate statistically significant results. Table 3 lists the simulation conditions used for each of the simulations. A total of 350 independent $\mathrm{MD}$ runs were performed.

Typical microstructures showing the GBs and broken bonds at the end of a simulation for the large-grain samples are displayed in figure 9 for simulations performed with maximum $\mathrm{GB}$ bond extension energies $E_{g b}$ between 0.255 and 0.648 of the matrix value $E_{\text {mat }}$. For $E_{g b} / E_{\text {mat }}=0.648$ (figure $9(b)$ ), a substantial portion of the main crack extends through matrix bonds starting near the location of the impact. Secondary cracks nucleate and grow mostly along grain boundaries. At a maximum energy of $E_{g b} / E_{\text {mat }}=0.497$ (figure $9(c)$ ), the main crack begins to grow transgranularly from the impact zone and then becomes increasingly intergranular as it progresses. The main crack shows several branches which are predominantly intergranular. At $E_{g b} / E_{\text {mat }}=0.366$ (figure $9(d)$ ), only a few short 
(a)

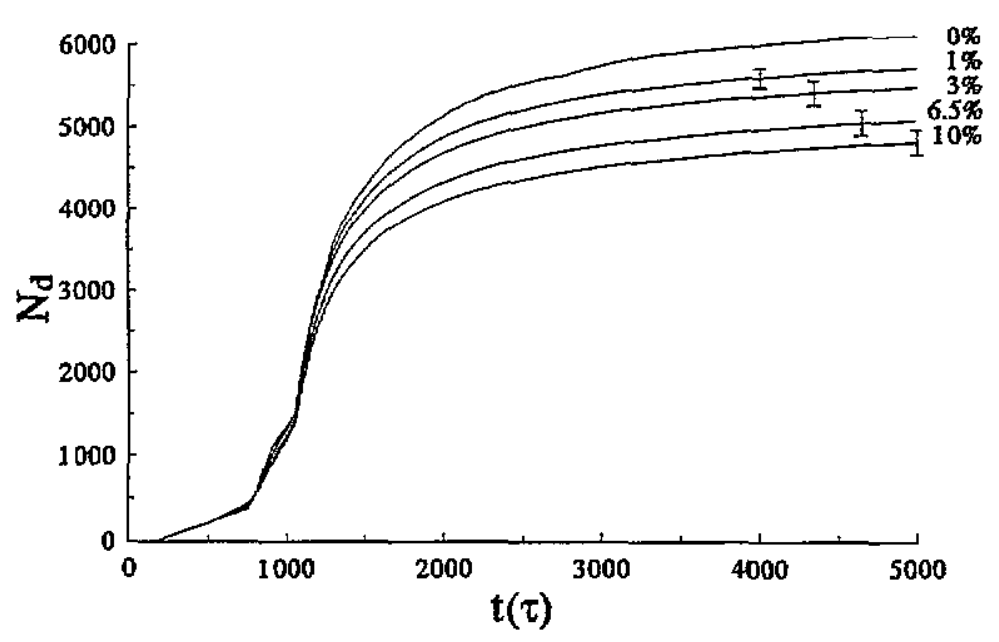

(b)

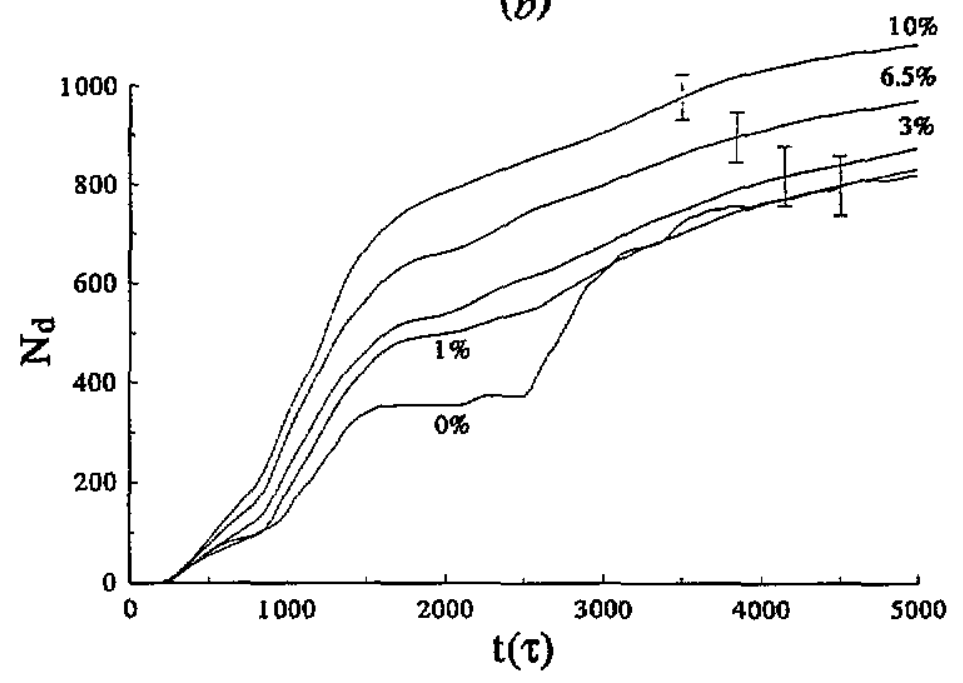

Figure 8. Number of bonds broken during simulation yersus time for simulations made at various initial defect levels: (a) $F_{\max }=0.750 \mathrm{~N} /$ particle; $(b) F_{\max }=0.375 \mathrm{~N} / \mathrm{particle}$.

straight transgranular cracks are observed and most of the cracking occurs in an intergranular mode with a significant fraction of the total number of GB bonds broken. Finally, when $E_{g b} / E_{\text {mat }}=0.255$ (figure $9(e)$ ), almost no transgranular cracking is observed and almost all of the GB bonds have been broken. At even lower values of $E_{g b} / E_{\text {mat }}$ the final configurations (not shown here) show that all the GB bonds are broken and virtually all of the matrix bonds are left intact. In other words, the polycrystalline material shatters into an ensemble of grain size particles.

The average number of broken matrix bonds and the average number of broken GB bonds at the end of each simulation are shown in figure $10(a)$ as a function of $E_{g b} / E_{\text {mat }}$ for the large-grain material subjected to impact pulse heights of $F_{\max }=0.3125 \mathrm{~N} /$ particle. 
Table 3. Number of runs at each test condition for the grain boundary simulations.

\begin{tabular}{|c|c|c|c|c|c|c|}
\hline \multirow[b]{2}{*}{$E_{\delta^{b}} / E_{\text {mat }}$} & \multicolumn{6}{|c|}{ Number of runs for the following $F_{\max }$ in N/Particle } \\
\hline & 0.2500 & 0.3125 & 0.3750 & 0.5000 & 0.6250 & 0.7500 \\
\hline \multicolumn{7}{|c|}{ Large-grain-size simulations } \\
\hline 0.0102 & & 10 & & & & 10 \\
\hline 0.0409 & & 10 & & & & 10 \\
\hline 0.0918 & & 10 & & & & 10 \\
\hline 0.1630 & 10 & 10 & 10 & 10 & 10 & 10 \\
\hline 0.2550 & & 10 & & & & 10 \\
\hline 0.3660 & & 10 & & & & 10 \\
\hline 0.4970 & 10 & 10 & 10 & 10 & 10 & 10 \\
\hline 0.6480 & & 10 & & & & 10 \\
\hline 0.8190 & & 10 & & & & 10 \\
\hline \multicolumn{7}{|c|}{ Small-grain-size simulations } \\
\hline $0.1020^{\circ}$ & & 10 & & & & \\
\hline 0.0409 & & 10 & & & & \\
\hline 0.0918 & & 10 & & & & \\
\hline 0.1630 & & 10 & & & & \\
\hline 0.2550 & & 10 & & & & \\
\hline 0.3660 & & 10 & & & & \\
\hline 0.4970 & & 10 & & & & \\
\hline 0.6480 & & 10 & & & & \\
\hline 0.8190 & & 10 & & & & \\
\hline
\end{tabular}

The number of broken GB bonds increases with decreasing GB bond strength, levelling off as $E_{g b} / E_{\text {mat }}$ is reduced to approximately 0.2 . At this plateau, the GB bonds have been sufficiently weakened such that virtually all GB bonds break by the end of the simulation. Additional decreases in $E_{g b} / E_{\text {mat }}$ have little effect. In contrast, the number of broken matrix bonds decreases with decreasing relative GB bond strength. The maximum rate of increase in the number of broken matrix bonds with increasing $E_{g b} / E_{\text {mat }}$ occurs over the same range in which the number of broken GB bonds decreases most quickly. The net effect of these trends in matrix and $\mathrm{GB}$ bonds is for the total number of broken bonds to increase with decreasing GB strength.

The dependence of the number of broken $G B$ and matrix bonds on the relative $G B$ strength $E_{g b} / E_{\text {mat }}$ for the large-grain samples impacted with a larger pulse height $F_{\max }=0.75 \mathrm{~N} /$ particle is shown in figure $10(b)$. As observed at the lower impact pulse height (see figure 10(a)), the number of broken GB bonds increases with decreasing GB strength, reaching a plateau at a larger $E_{g b} / E_{m u t}$ than observed at lower impact pulse heights. The number of broken matrix bonds in this case is much larger at all $E_{g b} / E_{\text {mut }}$ than in the low-impact-pulse-height simulations. This number decreases with decreasing $G B$ strength as in the low-impact-pulse-height case but does not saturate. The total number of bonds broken is significantly smaller (about 2800) for the weakest GB runs than for the case in which the GB strength is highest (about 6000).

The dependence of the number of broken matrix and GB bonds on $E_{g b} / E_{\text {mat }}$ is shown in figure $10(c)$ for the small-grain sample impacted with $F_{\max }=0.3125 \mathrm{~N} /$ particle. The curves of the number of broken matrix and GB bonds are qualitatively similar to those obtained for the large-grain samples subject to the same pulse height (figure 10(a)). The main difference between the coarse- and fine-grained samples is in the total number of broken GB bonds, 
(a)

(b)

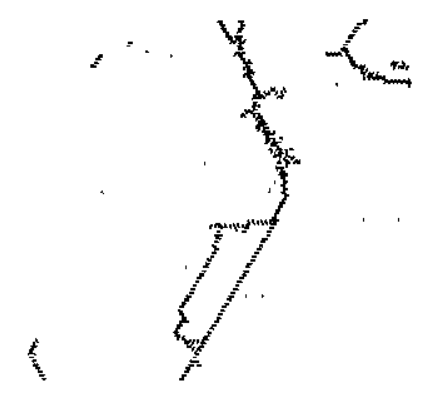

(c)

(d)
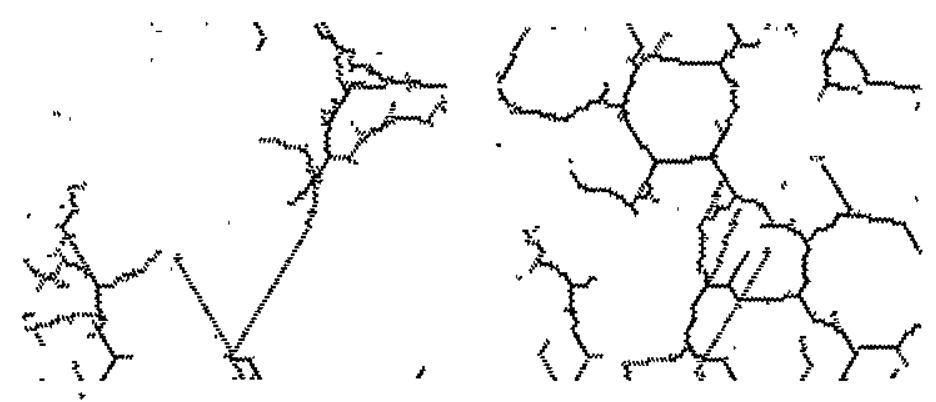

(e)

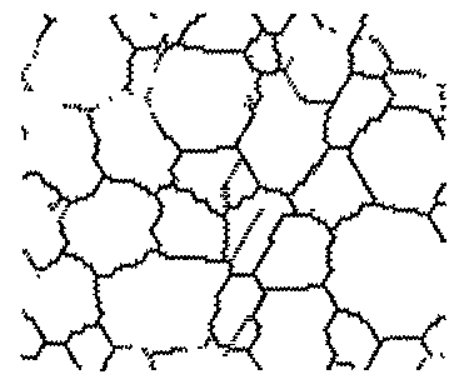

Figure 9. (a) Initial GB structure prior to impact and $(b)-(e)$ final distributions of broken bonds for a representative large-grain sample subjected to an impact of $F_{\max }=0.3125 \mathrm{~N} /$ particle: $(b)$ $E_{g b} / E_{m a t}=0.648 ;(c) E_{g b} / E_{m a t}=0.497 ;(d)_{g b} / E_{m a t}=0.366 ;(e) E_{g b} / E_{m a t}=0.255$. Only particles adjacent to the GB are plotted.

which is much larger in the fine-grained samples. This is simply attributable to the fact that there are many more GB bonds in the fine-grained sample and that, when $E_{g b} / E_{\text {mat }}$ is small, nearly all GB bonds break. In addition, the relative GB strength at which the number of broken GB bonds curve reaches its plateau is shifted to a lower value in the small-grain samples.

The dependence of the number of broken bonds on the impact pulse height is shown in figure 11 for the large-grain samples with $E_{g b} / E_{\text {mat }}=0.163$ and 0.497 . Figure $11(a)$ shows that the number of broken GB bonds increases with increasing pulse height up to a maximum value (all the GB bonds) and that the samples with weaker GB bonds exhibit more $\mathrm{GB}$ damage than do the stronger bonds at all impact levels. Matrix damage also increases with increasing pulse height, but the samples with the weakest GBs consistently 
(a)

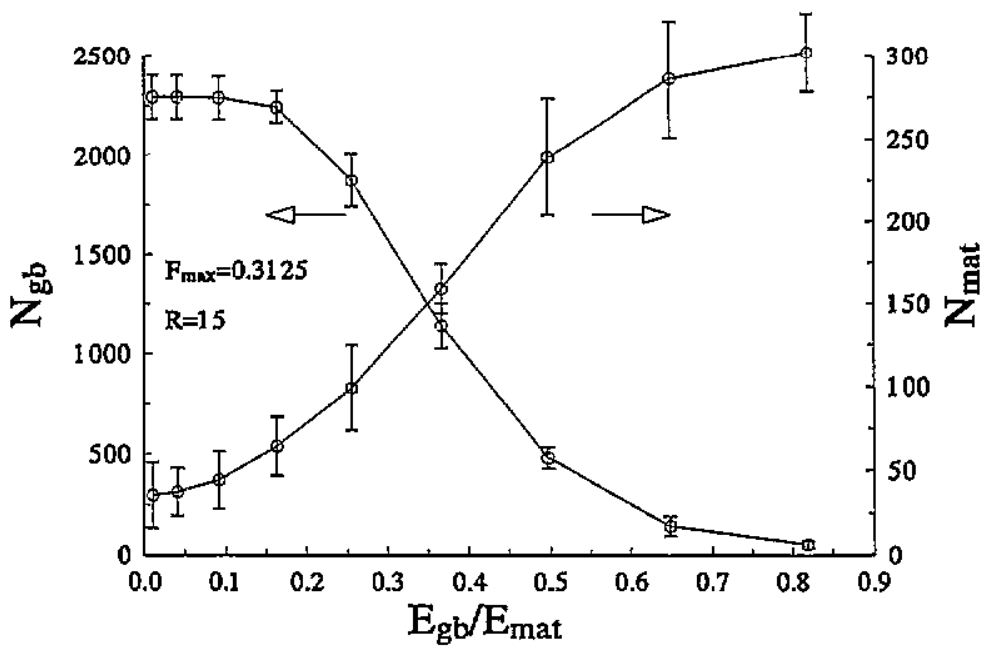

(b)

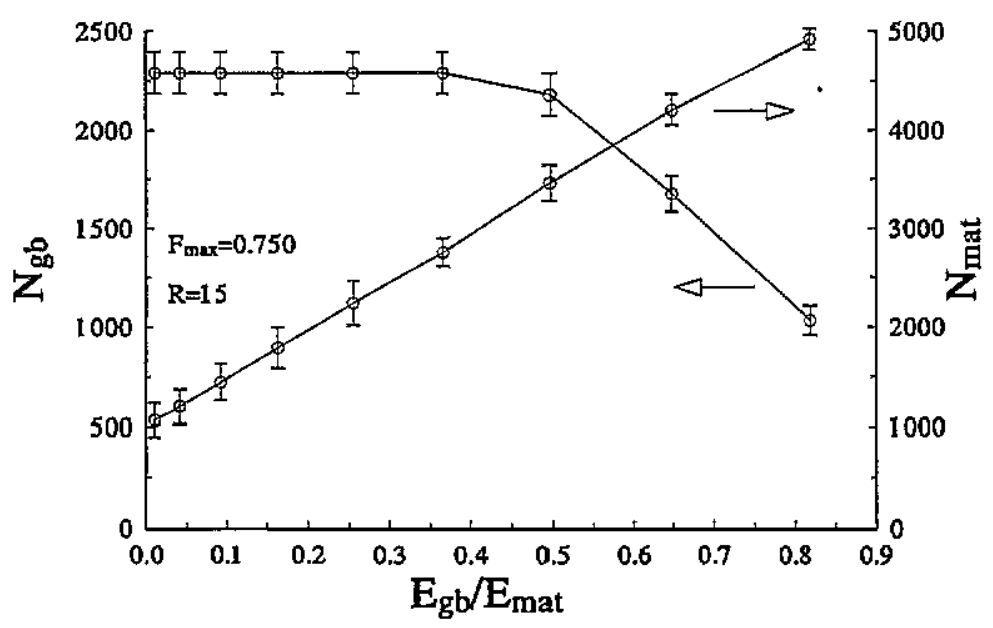

Figure 10. Total number of broken matrix bonds and broken GB bonds versus relative $G B$ strength: (a) $F_{\max }=0.3125 \mathrm{~N} /$ particle, large grain size; (b) $F_{\max }=0.750 \mathrm{~N} /$ particle, large grain size; $(c) F_{\max }=0.3125 \mathrm{~N} /$ particle, small grain size. Data points indicate an average of ten simulations. Error bars represent $\pm 1.0 \sigma$.

display less damage than do samples with stronger boundaries. The total damage (GB + matrix) is shown for these polycrystalline samples as a function of impact pulse height in figure $1 \mathbf{l}(b)$ together with the data for the perfect crystal (no GB) samples. At impact pulse heights lower than about $0.50 \mathrm{~N} /$ particle, the total damage observed increases as the GB strength decreases. At higher pulse heights, the trend is reversed, indicating that the total damage level is reduced as the GBs are made weaker.

The results presented in figures 9-11 show that the presence of weak GBs tends to increase the number of broken GB bonds and to decrease the number of broken matrix bonds. Depending on the height of the impact pulse applied, the total number of broken 
(c)

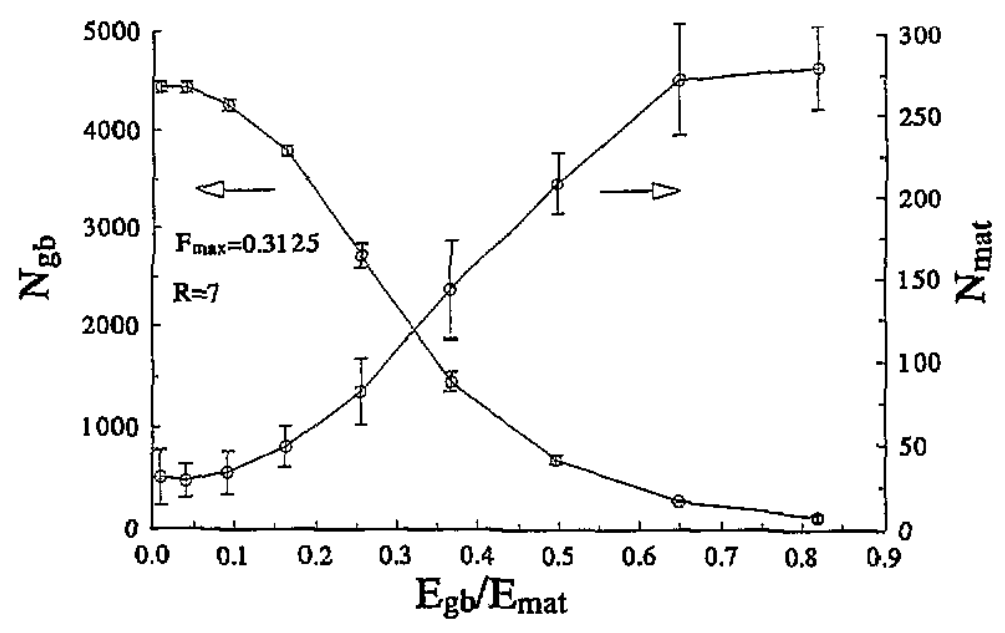

Figure 10. (Continued)

bonds may be either increased or decreased due to the presence of weak boundaries. In order to gain some insight into the mechanisms by which the weak boundaries influence the propagation of cracks, it is instructive to look at the time dependence of damage formation for both matrix and GB bonds. Figure 12 presents the numbers of broken matrix and broken GB bonds as functions of time for the large-grain samples subjected to a relatively small impact of $F_{\max }=0.3125 \mathrm{~N} /$ particle. Different curves within the plot represent different values of GB strength expressed as $E_{g b} / E_{\text {mat }}$. As shown in figure $12(a)$, GB bonds begin to break near the start of the impact and undergo an initial increase in the bond-breaking rate, followed by a decrease in the damage rate as the total number of broken bonds approaches the maximum value. As the strength of the boundary bonds is reduced, the curves asymptote earlier. The total number of broken matrix bonds shows the opposite trend (figure $12(b)$ ). As the boundaries are weakened, the number of broken matrix bonds increases more slowly. In addition, the maximum number of broken matrix bonds is decreased as the boundary bond strength is reduced. The acceleration in damage accumulation observed at $t \approx 2500 \tau$ in the initially defect-free sample (see figure $4(a)$ ) is also present here, but only for the relatively strong-GB cases. As the GB bond strength is reduced, this sudden acceleration becomes less detectable.

The time dependence of the damage accumulation is shown in figures $12(c)$ and $12(d)$ for the highest-amplitude-impact simulations $\left(F_{m a x}=0.75 \mathrm{~N} /\right.$ particle $)$. The behaviour of the broken GB bond curves is similar to that observed in the low-impact case; however, due to the stronger impact, the damage level reaches its maximum early in the simulation even for relatively strong boundaries. The number of broken matrix bonds displays a similar time dependence to that observed for the initially defect-free sample (see figure $4(b)$ ). The damage rate appears insensitive to GB strength before $t \approx 750 \tau$. At this time the matrix damage accumulation rate increases and the magnitude of the increase is largest for strongboundary samples and smallest for the weaker-boundary samples. The maximum number of broken matrix bonds decreases as the relative GB strength decreases. The second increase in the matrix damage accumulation rate observed at $t \approx 1000 \tau$ in the initially defected-free material (see figure $4(b)$ ) is present in the matrix bond data for samples with strong GB 
(a)

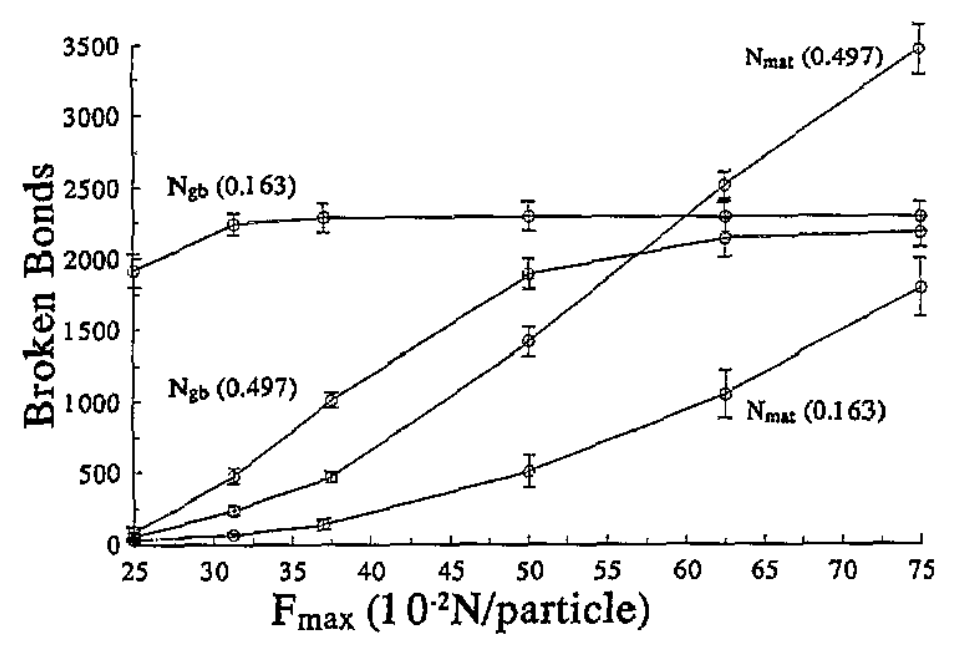

(b)

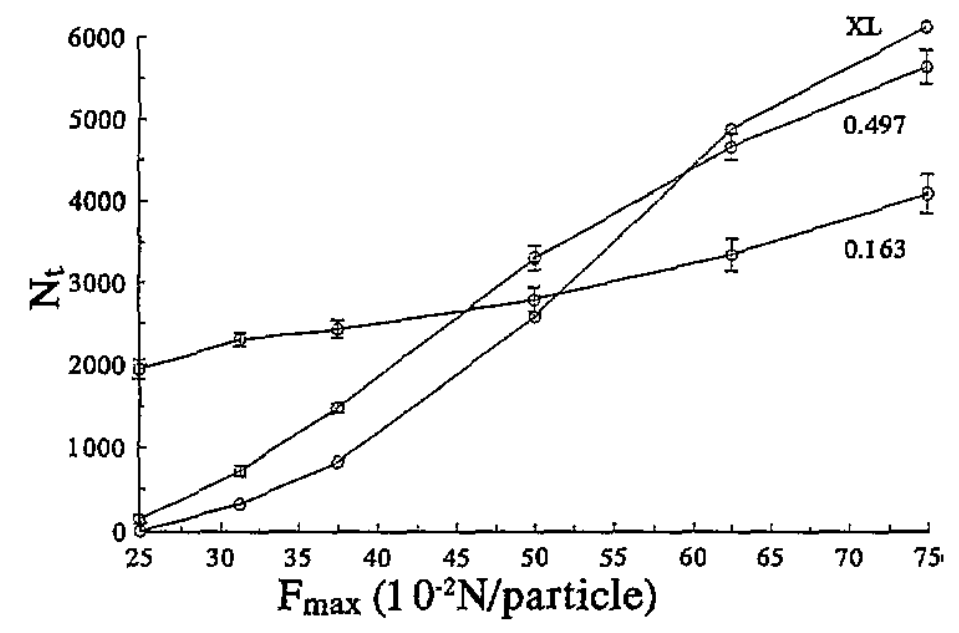

Figure 11. Number of broken bonds versus impact pulse height: (a) broken GB and matrix bonds for samples with $E_{g b} / E_{m a t}=0.163$ and 0.497 ; (b) total number of broken bonds compared with the perfect crystal data (line XL).

bonds (figure $12(d)$ ). As the bonds are weakened, the change in damage rate at this time becomes less pronounced.

\section{Discussion}

\subsection{Perfect material}

In order to understand the effects of pre-existing microcracks and GBs on damage accumulation and fracture of brittle solids, we initially focus on the perfect material in order to establish a baseline. In both the high- and low-amplitude impact simulations (figures 2 and 3 ), the significant features are the formation of an initial $X$-shaped cracking pattern and the nucleation of independent damage regions.

The formation and propagation of the $X$ crack in the low-impact simulation (figure 
2) have been described in detail in a previous paper [17]. This crack results from the propagation of the initial impact wave which extends from the impact region into the buik of the material. This crack is driven by the superposition of the impact pulse waves emanating from the edges of the impact zone prior to any wave reflection from the free surfaces. The cracks which nucleate on the outside of the $\mathrm{X}$ crack at $t=1000 \tau$ are attributable to the superposition of the release waves formed when the applied load is removed and the initial impact waves reflected back from the vertical free surfaces (note that the sign of this wave changes from compression to tension upon reflection). The cracks which form near the top corners of the sample at $t=2500 \tau$ also result from the superposition of multiply reflected elastic waves. The first reflection of the incident wave from the top surface takes place at roughly $t=900 \tau$. Because the amplitude of the incident wave decreases by the time that it reaches this surface, it has insufficient energy upon reflection to break the bonds in this region. Only after several multi-reflected waves (scattered from each of the surfaces) have interfered constructively is sufficient energy deposited in this location to break these bonds.

In the high-impact-pulse-height case, the damage also begins with the formation of an $\mathrm{X}$-shaped crack (figure 3 ) but, unlike the low-impact simulation, this crack is immediately flanked by two additional cracks running into the material parallel to the branches of the $\mathrm{X}$. These cracks begin on the impacted surface at the edges of the impact zone. Whereas the central $\mathrm{X}$ crack is comprised mostly of broken horizontal bonds, these additional cracks contain broken bonds which lie at $60^{\circ}$ to the horizontal. The orientation of these bonds suggests that the material immediately beneath the impact zone is being sheared away from the free standing material on either side of it. The acceleration in cracking seen at $t=1000 \tau$ corresponds to cracks which form near the top surface of the sample. These cracks result from the large-amplitude tensile wave which forms from the first reflection of the incident (compressive) wave. The curvature of this crack front (figure $3(c)$ ) corresponds to the leading edge of the reflected tensile wave. Unlike the low-impact case, the energy of the reflected wave is sufficient in this case to cause damage immediately even in the absence of superposition from other reflected wave fronts.

\subsection{Microcracked material}

While an increase in the total number $N_{t}$ of broken bonds with increasing initial microcrack density is to be expected, the nature of the damage accumulated during the simulation as indicated by $N_{d}$ displays an interesting feature. At low impact pulse heights, $N_{d}$ increases with increasing initial microcrack density but, at higher impact pulse heights, $N_{d}$ decreases as the initial microcrack density increases (see figure 8 ). When the pulse height is low, the pre-existing microcracks accentuate cracking by weakening the material, thereby allowing more damage to develop in the wake of the initial stress wave near the beginning of the simulation. The breaking of additional bonds stems simply from the fact that, since a pre-broken bond supports no load in tension, then this load must be redistributed amongst the neighbouring bonds. The increased stress makes it more likely that one or more of these bonds will be stretched beyond its breaking limit, causing those bonds to fail. This mechanism is important at low impact levels where most of the sample is not subjected to stresses strong enough to break bonds in the perfect material. This simple picture explains the nucleation of new cracks in the vicinity of pre-broken bonds and the alteration of the crack propagation paths to link up with existing defects. The increased level of damage accompanying an increased number of pre-broken bonds is consistent with expectations based on the increase in the stress intensity factor associated with adjacent cracks compared with an individual crack. This relation has also been demonstrated via quasi-static computer 
simulations of brittle failure conducted by Srolovitz and Beale [20]. In this work, the failure stress as well as Young's modulus were found to be decreasing functions of initial defect concentration.

(a)

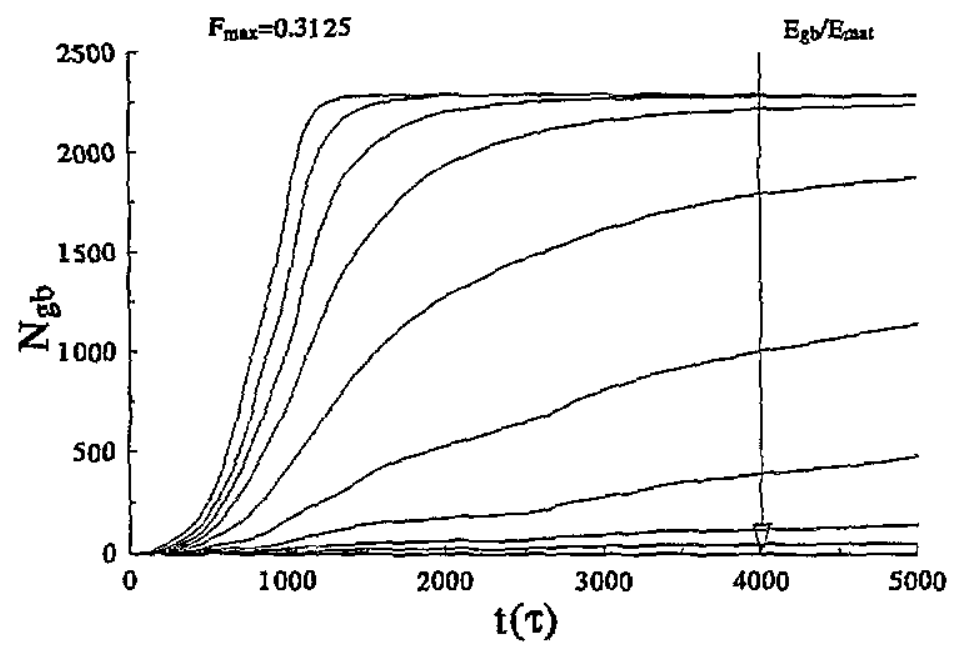

(b)

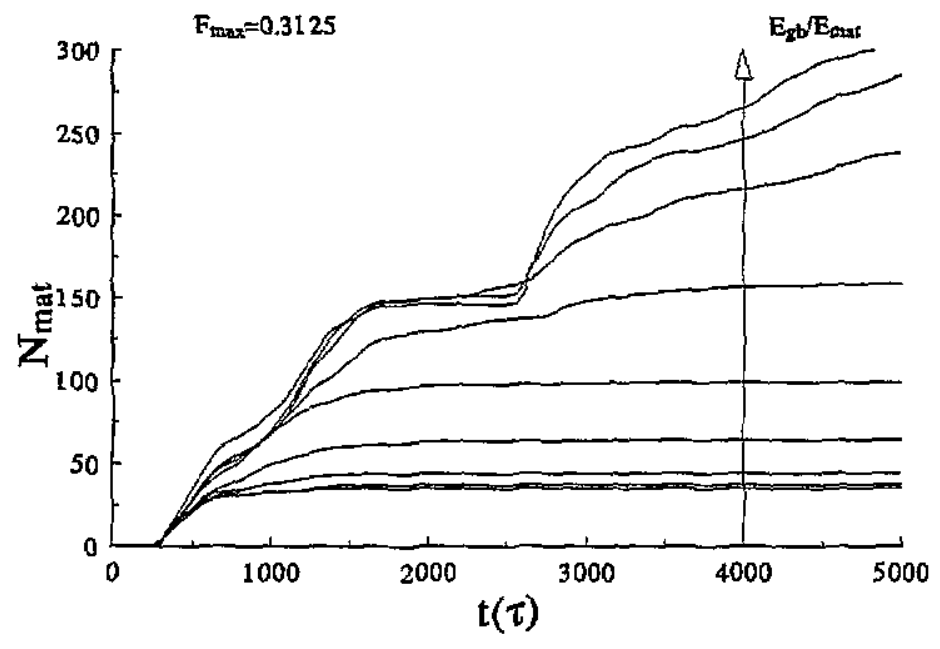

Figure 12. Number of broken bonds versus time for large-grain samples: (a) $F_{\max }=0.3125$ $\mathrm{N} /$ particle, GB bonds; (b) $F_{\max }=0.3125 \mathrm{~N} /$ particle, matrix bonds; (c) $F_{\max }=0.750 \mathrm{~N} /$ particle, GB bonds; (d) $F_{\max }=0.750 \mathrm{~N} /$ particle, matrix bonds.

At high impact pulse heights, on the other hand, the presence of pre-existing microcracks tends to spare much of the material from damage which forms in the simulations of initially defect-free samples. In this case, the damage which forms near the start of the simulations is insensitive to the presence of the microcracks because the initial impact is strong enough to fracture a large number of bonds even in the absence of these cracks. The damage which forms later, as a result of the reflection of the incident wave from the top surface, 
(c)

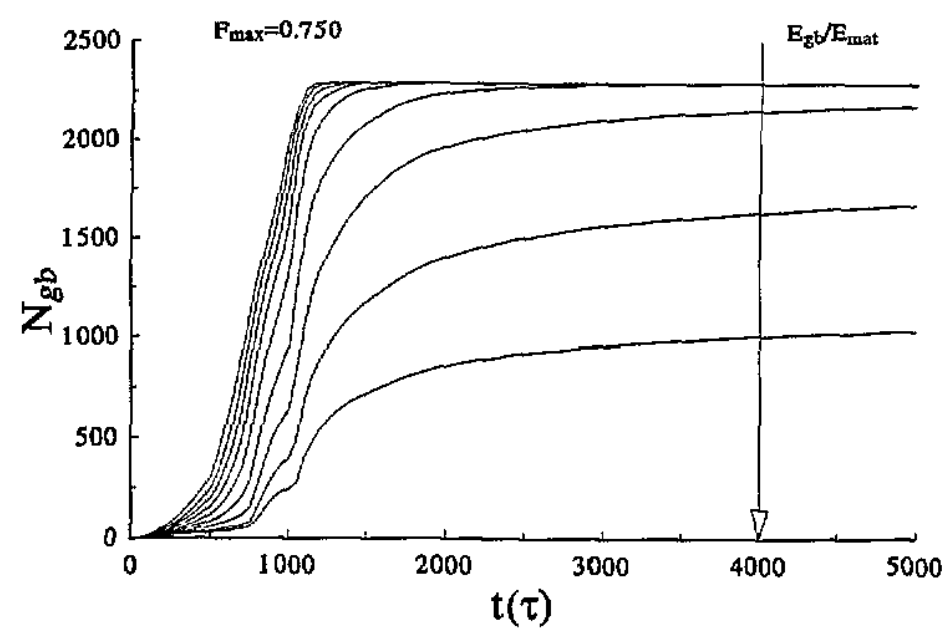

(d)

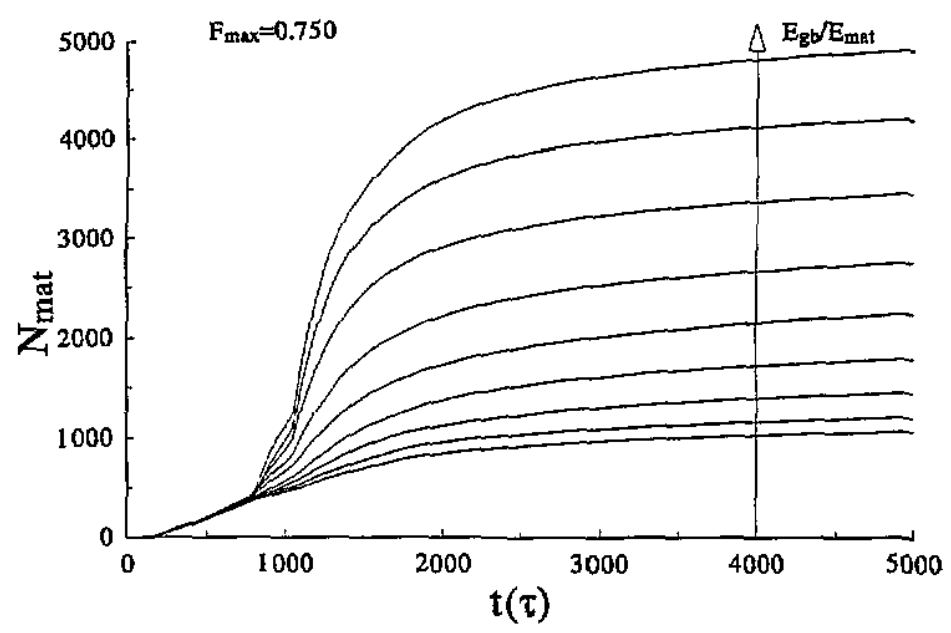

Figure 12. (Continued)

however, is impeded because the reflected (tensile) wave is scattered or dispersed by the field of pre-existing microcracks. This effect can best be understood by considering the influence of pre-broken bonds on the transport of elastic waves through the material. Since the compressive behaviour of a snapped bond is identical with that of one that is intact (see equation (2)), the only influence exerted by the broken bonds is on tensile waves or the tensile components of more complex waves. Since a snapped bond cannot support a tensile stress, this wave front is in part reflected (as it is from a free surface) and in part scattered to either side of the broken bond. This combined effect can be to convert part of a tensile wave front into compressive waves propagating in the opposite direction, defocusing and hence decreasing the wave amplitude, and thereby to teduce the number of cracked bonds formed there. 
Evidence of the retarding effect of microcracks on reflected waves is also observed in the low-impact simulations, but to a smaller extent. Comparison of figures $2(f)$ (initially defect free) and 5 ( $1 \%$ initial defect level) shows that the regions of damage formed in the upper corners of the defect-free sample due to multiple reflections are largely missing in the microcracked sample. In addition, the acceleration in cracking rate which accompanies the nucleation of these regions $(t=2500 \tau)$ is not observed in the simulations of the microcracked samples. In contrast with the high-impact case, however, when the pulse height is small, the retarding effect is insufficient to compensate for the increased crack rate described above.

A reduction in damage accumulation in low-strain-rate fracture experiments due to the presence of pre-existing microcracks has been suggested by Kuszyk and Bradt [22] and by Evans and Faber [23]. In their microcrack reinforcement model the stress concentration near the tip of the moving macrocrack is diminished by the growth of the isolated microcracks ahead of it. So long as the microcracks do not grow sufficiently to link up, the overall effect is to slow the progress of the macrocrack. For larger concentrations of microcracks, coalescence together with catastrophic failure becomes more likely [24]. Although the present experiments show some isolated regions in which damage is apparently reduced, the net result of pre-existing microcracks is to increase the total number of bonds broken at the end of the simulation. Nonetheless, our results do show that the microcracks are able to stop the growth of one of the two main cracks (one arm of the X) observed in the microcrack-free sample, in agreement with the static picture.

\subsection{Polycrystalline material}

As described above, a reduction in the relative $\mathrm{GB}$ bond strength produces an increase in the number of broken GB bonds and a decrease in the number of matrix bonds which fracture during the impact loading simulations. Depending on the impact pulse height, the total number of broken bonds (matrix $+G B$ ) may either increase or decrease with $G B$ bond strength (see figure $11(b)$ ). The reason for the increase in the number of broken $G B$ bonds as GB bond strength is reduced is obvious: weaker bonds are easier to break. A similar transition from transgranular to intergranular fracture with decreasing GB bond strength has been observed with low-strain-rate (i.e. quasi-static) simulations of brittle samples in tension [25].

The reduction in the number of broken matrix bonds with decreasing GB bond strength is attributable to the fact that, when a GB is broken, tensile waves cannot be transferred through it into the grain interior (i.e. the matrix). Since the GB bonds differ from matrix bonds only in their maximum elongation to fracture it is clear that the boundaries may influence only the passage of elastic waves after they have fractured and that only the tensile component of the waves may be affected. The fractured boundary behaves like a free surface, reffecting a large portion of the tensile wave. There is, however, a significant difference between the wave reflection behaviour at free surfaces and that at GBs. At a free surface, a compressive wave is reflected and inverted to produce a tensile wave, which can readily cause bond breakage. At a GB however, the compressive waves pass unreflected because the GB bonds support compression even if fractured. It is a fact that broken GBs reflect tensile waves and not compressive waves that allow them to divert the most damaging waveforms away from the grain interiors. The influence of these GBs on the propagation of tensile-wave-induced damage may be readily seen in figures $12(b)$ and $12(d)$. These plots show that the sudden accelerations in bond breakage which were associated with the reflection of compressive waves from the free surfaces in the initially defect-free samples 
are greatly diminished in the samples containing weak GB bonds.

The two important variables in a study of the effects of GBs on the impact damage resistance of a polycrystalline material are GB strength and grain size. While we were able to study exhaustively the effect of GB strength, our data on the grain size effect is significantly more limited. Comparison of figures $10(a)$ and $10(c)$ reveals that, at least at the low impact pulse height $F_{\max }=0.3125 \mathrm{~N} /$ particle, the small-grain samples suffer more damage than the larger-grain samples do for all GB bond strengths tested. This suggests simply that, in the regime where most of the damage occurs along the GBs, more damage will occur as the relative number of GB bonds is increased. Experimental studies of the effect of GBs on impact fracture have been conducted by Ritter and co-workers [7-9], but these experiments were performed only at a single impact strength. In these investigations, coarse-grained $\mathrm{SiN}$ and $\mathrm{Al}_{2} \mathrm{O}_{3}$ were found to be more impact resistant (as measured by post-impact fracture strength) than fine-grained samples of the same materials, in agreement with our preliminary results for GBs at all levels of embrittiement.

Finally, we note that, in the present study, we have focused upon the total number of broken bonds as a measure of the damage in the material. Other measures of damage (such as distance that it is possible to propagate a crack or tendency for spallation at the back side) might not lead to the same conclusions.

\section{Conclusions}

A modified MD method was introduced to describe the dynamic fracture and damage accumulation in impact-loaded brittle solids with realistic microstructural features. In the present study, we have examined the influence of pre-existing microcracks and GBs on dynamic fracture.

Pre-existing microcracks were found to increase the total damage within a material for all combinations of impact pulse height and microcrack concentration studied. The damage accumulated due to the impact itself, however, is found to increase with increasing concentration of pre-existing microcracks at low impact pulse heights and to decrease with increasing concentration at higher impact levels. Therefore, microcracks may either enhance cracking by providing weaker crack paths or retard it by reflecting and dissipating tensile elastic waves.

The presence of weak GBs in a polycrystalline material was found to enhance intergranular fracture while diverting cracks away from grain interiors. Weak GBs lead to the fragmentation of the polycrystalline material into grain size powders. The number of broken GB bonds increases and the number of broken matrix bonds decreases as the GB bond strength is reduced. At low impact pulse strengths, the total number of broken bonds increases as the GB bonds are weakened, but at high pulse strengths the total number of broken bonds is actually reduced as the GB bond strength is reduced. Increasing impact strength shifts the fracture mode from intergranular to transgranular.

The principal infiuence of both microcracks and GBs appears to be their ability to scatter elastic waves propagating through the material.

\section{Acknowledgments}

This research was supported by the Advanced Research Projects Agency and the Office of Naval Research under contract N00014-91-J-4019. 


\section{References}

[1] Kunze H D and Meyer L W (eds) 1988 Proc. Int. Conf. on Impact Loading and Dynamic Behaviour of Materials (Darmstadt: Deutsche Gesellschaft für Metallkunde)

[2] Lackey W J, Stinten D P, Cerny G A, Fehrenbacher L L and Schaffhauser A C 1984 Oak Ridge National Laboratory Repurt ORNL/TM-8959

[3] Hendricks R C and McDonald G 1982 Ceram. Eng, Sci. Proc. 3737

[4] Kamo R, Wood M and Geary W 1983 Ceramics for High Performance Applications. III, Proc. 6th Army Materials Technology Conf. ed E Lenoe, R N Katz and J J Burke (New York: Plenum) p 187

[5] Gilath I, Eliezer S and Gazit Y 1991 J. Mater. Sci. 262023

[6] Mackawa I, Shin H-S and Hiyata H 1990 Key Eng. Mater. 51173

[7] Ritter J E 1991 Ceram. Int. 17165

[8] Ritter J E, Choi S R, Jakus K, Whalen P J and Rateick R G Jr 1991 J. Mater. Sci. 265543

[9] Brededer K, Ritter J E and Jakus K $1988 \mathrm{~J}$. Am. Ceram. Soc. 711154

[10] Louro L H and Meyers M A 1989 J. Mater. Sci. 242516

[11] Holian K S and Burkett M W 1987 Int. J. Impact Eng. 5333

[12] Liaw B M, Kobayashi A S, Emery A F and Du J J 1986 Fracture Mechanics of Ceramics (Composites, Impact, Statics, and High Temperature Phenomena) vol 7, ed R C Bradt, A G Evans, D P H Hasselman and F F Lange (New York: Plenum) p 187-96

[13] Holian B L 1987 Phys. Rev. A 363943

[14] Holian B L, Voter A F, Wagner N H, Ravelo R J, Chen S P, Hoover W G, Hoover C G, Hammerberg J E and Dontje T D 1991 Phys. Rev. A 432655

[15] Abraham F F and Rudge W E 1989 Chem. Phys. 129263

[16] Daw M S and Baskes M 1984 Phys. Rey, B 296443

[17] Smith R W and Srolovitz D J 1994 Modelling Simul. Mater. Sci. Eng. 21153

[18] Yang W H, Srolovitz D J, Hassold G N and Anderson M P 1990 Microstructural effects in the fracture of brittle materials Simulation and Theory of Evolving Microstructures ed M P Anderson and A D Rollett (Warrendale, PA: The Metallurgical Society) pp 277-84

[19] Yang W H unpublished results

[20] Srolovitz D J and Beale P D 1988 J. Am. Ceram. Soc. 71362

[21] Grest G S, Anderson M P and Srolovitz D J 1988 Phil. Mag. B 59293

[22] Kuszyk J A and Bradt R C $1973 \mathrm{~J}$. Am. Ceram. Soc. 56420

[23] Evans A G and Faber K T 1984 J. Am. Ceram, Soc. 68255

[24] Petzow G, Telle R and Danzer R 1973 Mater. Characterization 26289

[25] Srolovitz D J, Yang W H, Najafabadi R, Wang H Y and LeSar R 1992 Materials Interfaces, Atomic-Level Structure and Properties ed D Wolf and S Yip (New York: Chapman and Hall) p 691-701 